\title{
NOTE ON THE APPROXIMATE VALUES OF BESSEL'S FUNCTIONS FOR LARGE ARGUMENTS.
}

\author{
By Louis Cohen.
}

In many physical problems we frequently meet with Bessel's functions of a complex argument where the real part of the argument is a large quantity. Under these conditions the Bessel functions can be expressed in terms of exponential functions. Thus:

$$
\left.\begin{array}{ll}
J_{0}(i x)=\frac{e^{x}}{\sqrt{2 \pi x}}, & J_{1}(i x)=\frac{i e^{x}}{\sqrt{2 \pi x}} \\
G_{0}(i x)=\frac{\sqrt{\pi} e^{-x}}{\sqrt{2 x}}, & G_{1}(i x)=\frac{-i \sqrt{\pi} e^{-x}}{\sqrt{2 x}}
\end{array}\right\}
$$

$J_{0}$ and $J_{1}$ represent the Bessel functions of the first kind and of the zero and first order, respectively. $G_{0}$ and $G_{1}$ represent functions which are combinations of the Bessel functions of the first and second kinds, and have the property that the functions approach zero when the arguments become very large. The values of $G_{0}(i x)$ and $G_{1}(i x)$ in terms of the Bessel functions of the first and second kinds are given by the following equations: ${ }^{1}$

$$
\left.\begin{array}{l}
G_{0}(i x)=(\log 2-\gamma) J_{0}(i x)-Y_{0}(i x) \\
G_{1}(i x)=(\log 2-\gamma) J_{1}(i x)-Y_{1}(i x)
\end{array}\right\}
$$

$Y_{0}$ and $Y_{1}$ are the Bessel functions of the second kind and $\gamma$ is Euler's constant $(\gamma=0.5772 \mathrm{I})$.

The values of the Bessel functions as given by equation (I) are usually obtained by the aid of the theory of functions of a complex variable; the proof is very laborious and difficult.

${ }^{1}$ See Gray and Mathews: Treatise on Bessel Functions, pp. 68, 91. 
I have worked out a simple demonstration, which, though it is not rigorous, will, I trust, be of interest to physicists.

When the real part of the argument is positive we can express the Bessel function of the first kind in the following form: ${ }^{2}$

where

$$
J_{n}(y)=\sqrt{\frac{2}{\pi y}}\left[P \cos \left\{\frac{(2 n+I)}{4} \pi-y\right\}+Q \sin \left\{\left(\frac{2 n+I}{4}\right) \pi-\right\}\right]
$$

and hence

$$
\begin{gathered}
P=I-\frac{\left(4 n^{2}-I\right)\left(4 n^{2}-9\right)}{2 !(8 y)^{2}}+\ldots \\
Q=\frac{4 n^{2}-I}{8 x}-\frac{\left(4 n^{2}-I\right)\left(4 n^{2}-9\right)\left(4 n^{2}-25\right)}{3 !(8 y)^{3}}+\ldots
\end{gathered}
$$

now

$$
\begin{aligned}
& J_{0}(y)=\sqrt{\frac{2}{\pi y}}\left[P \cos \left(\frac{\pi}{4}-y\right)+Q \sin \left(\frac{\pi}{4}-y\right)\right] \\
& J_{1}(y)=\sqrt{\frac{2}{\pi y}}\left[P \cos \left(\frac{3 \pi}{4}-y\right)+Q \sin \left(\frac{3 \pi}{4}-y\right)\right]
\end{aligned}
$$

$$
\left.\begin{array}{c}
\cos \left(\frac{\pi}{4}-y\right)=\frac{\mathrm{I}}{\sqrt{2}}(\cos y+\sin y) \\
\sin \left(\frac{\pi}{4}-y\right)=\frac{\mathrm{I}}{\sqrt{2}}(\cos y-\sin y) \\
\cos \left(\frac{3 \pi}{4}-y\right)=\frac{\mathrm{I}}{\sqrt{2}}(-\cos y+\sin y) \\
\sin \left(\frac{3 \pi}{4}-y\right)=\frac{\mathrm{I}}{\sqrt{2}}(\cos y+\sin y) \\
\therefore J_{0}(y)=\frac{\mathrm{I}}{\sqrt{\pi y}}[(P+Q) \cos y+(P-Q) \sin y] \\
J_{1}(y)=\frac{\mathrm{I}}{\sqrt{\pi y}}[(Q-P) \cos y+(P+Q) \sin y]
\end{array}\right\}
$$

If $y$ is very large we can put $P=\mathrm{I}$ and neglect $Q$ as compared with $P$, and we shall have

$$
\begin{aligned}
& J_{0}(y)=\frac{\mathrm{I}}{\sqrt{\pi y}}\{\cos y+\sin y\} \\
& J_{1}(y)=\frac{\mathrm{I}}{\sqrt{\pi y}}\{-\cos y+\sin y\}
\end{aligned}
$$

${ }^{2}$ Gray and Mathews: Bessel Functions, p. 40. 
We also have

$$
\begin{aligned}
& \cos y=\frac{\mathrm{I}}{2}\left(e^{i y}+e^{-i y}\right) \\
& \sin y=\frac{-i}{2}\left(e^{i y}-e^{-i y}\right)
\end{aligned}
$$

put now $y=i x$, and we get

$$
\begin{aligned}
& J_{0}(i x)=\frac{\mathrm{I}}{2 \sqrt{\pi i x}}\left\{(\mathrm{I}-i) e^{-x}+(\mathrm{I}+i) e^{x}\right\} \\
& J_{1}(i x)=\frac{\mathrm{I}}{2 \sqrt{\pi i x}}\left\{-(\mathrm{I}+i) e^{-x}+(i-\mathrm{I}) e^{x}\right\}
\end{aligned}
$$

Neglecting $e^{-x} \quad$ as compared with $e^{x}$, we get

$$
\left.\begin{array}{l}
J_{0}(i x)=\frac{\mathrm{I}}{2 \sqrt{\pi i x}}(\mathrm{I}+i) e^{x}=\frac{\mathrm{I}}{\sqrt{2 \pi x}} e^{x} \\
J_{1}(i x)=\frac{\mathrm{I}}{2 \sqrt{\pi i x}}(i-\mathrm{I}) e^{x}=\frac{i}{\sqrt{2 \pi x}} e^{x}
\end{array}\right\}
$$

The values of the $G$ functions can be obtained in the following way: There exists a well-known relation between the Bessel functions of the first and second kinds, which is as follows:

$$
J_{1}(i x) Y_{0}(i x)-J_{0}(i x) Y_{1}(i x)=\frac{I}{i x}
$$

and if we substitute in equation (6) the values $J_{0}$ and $J_{1}$ as given by equation (5) we get

$$
\begin{aligned}
& \frac{e^{x}}{\sqrt{2 \pi x}}\left\{i Y_{0}(i x)-Y_{1}(i x)\right\}=\frac{\mathrm{I}}{i x} \\
& \text { or } i Y_{0}(i x)-Y_{1}(i x)=\frac{\mathrm{I}}{i} \sqrt{\frac{2 \pi}{x}} e^{-x}
\end{aligned}
$$

From equation (2) we can get the following relation:

$$
i G_{0}(i x)-G_{1}(i x)=(\log 2-\gamma)\left(i J_{0}(i x)-J_{1}(i x)\right)-i Y_{0}(i x)+Y_{1}(i x)
$$

Since, however, $J_{1}(i x)=i J_{0}(i x)$ (see equation 5$)$ we get 
or

$$
\begin{gathered}
i G_{0}(i x)-G_{1}(i x)=Y_{1}(i x)-i Y_{0}(i x)=-\sqrt[I]{\frac{2 \pi}{x}} e^{-x} \\
i G_{0}(i x)-G_{1}(i x)=i \sqrt{\frac{2 \pi}{x} e^{-x}}
\end{gathered}
$$

The values of $G_{0}$ and $G_{1}$ as given by equation (I) will satisfy the above equation; introducing the values we get

$$
\left(i \sqrt{\frac{\pi}{2 x}}+i \sqrt{\frac{\pi}{2 x}}\right) e^{-x}=i \sqrt{\frac{2 \pi}{x}}
$$

which is identical with (8), and hence it is reasonable to suppose that the values of $G_{0}$ and $G_{1}$ as given by equation (I) are the proper solutions.

Washington, August 24, I908. 